\title{
The Role of Prophylactic Lymph Node Dissection in Patients with Intrahepatic Cholangiocarcinoma
}

\section{Daisuke Satoh*, Hiroyoshi Matsukawa, Hiroyuki Araki and Shigehiro Shiozaki}

Department of Gastroenterological Surgery, Hiroshima City Hiroshima Citizens Hospital, 7-33 Motomachi, Naka-ku, Hiroshima, Japan

*Address for Correspondence

Daisuke Satoh, MD, Department of Gastroenterological Surgery, Hiroshima City Hiroshima Citizens Hospital, 7-33 Motomachi, Naka-ku, Hiroshima, Japan, Tel: +81-82-221-2291; Fax: +81-82-223-5514; E-mail: ddds4863@ gmail.com

Submission: 19 September, 2016

Accepted: 20 October, 2016

Published: 27 October, 2016

Copyright: (c) 2016 Satoh D, et al. This is an open access article distributed under the Creative Commons Attribution License, which permits unrestricted use, distribution, and reproduction in any medium, provided the original work is properly cited. impact on survival of prophylactic LND and preoperative risk factors for histologic al lymph node metastasis (LNM) in a cohort of 49 patients with ICC of the mass-forming type, who underwent curative resection between 1995 and 2013.

Results: There were no differences in both disease-free survival (DFS) and overall survival (OS) between the patients without LND and the patients with LND who did not have LNM. Multivariate analysis revealed that serum CA19-9 level $>150 \mathrm{U} / \mathrm{mL}$ and $\mathrm{LN}$ size $>5 \mathrm{~mm}$ on preoperative computed tomography (CT) were the significant preoperative risk factors for LNM. None of the patients with LN size $<5$ $\mathrm{mm}$ on preoperative CT and serum CA19-9 level $\leq 150 \mathrm{U} / \mathrm{mL}$ had LNM.

Conclusions: Prophylactic LND did not significantly improve DFS or OS in the present study. Regional LND could be omitted for patients with $\mathrm{LN}$ size $<5 \mathrm{~mm}$ in preoperative CT and serum CA 19-9 level $\leq 150 \mathrm{U} /$ $\mathrm{mL}$ who are unlikely to have LNM.

\section{Introduction}

Although intrahepatic cholangiocarcinoma (ICC) is a relatively rare tumor, accounting for $10-15 \%$ of all primary liver cancer $[1,2]$ the incidence of this disease has steadily increased in both Japan and throughout the world [3-5]. The prognosis of ICC is dismal, with an estimated median survival of only 18-19 months and an estimated 5-year survival rate between 25-40\% [6-8]. Curative hepatic resection is the only effective treatment for ICC. Meanwhile, the role of lymph node dissection (LND) is still unclear, despite lymph node metastasis (LNM) having been reported to be a significant risk factor for poor outcome [9-11]. Some institutions advocate the routine performance of LND for ICC [12]. On the other hand, other institutions consider selective LND and limited application of LND to be reasonable because the prognosis of patients with LNM is poor even if systematic LND is performed $[13,14]$. The efficacy of LND is still controversial regarding both treatment for ICC patients with LNM, and prophylactic treatment for ICC patients without LNM. It is desirable for routine and systematic LND to be avoided if possible, especially in the case of prophylactic LND performed in patients without LNM, because performance of LND has been shown to cause increased operative morbidity compared with patients who do not undergo LND $[15,16]$.

Another problem is that preoperative imaging assessment of LNM is quite difficult [17-19], although

LNM is considered to be the most important prognostic factor for

survival of patients with ICC. Accurate preoperative determination of LNM status is important [9-11], considered that routine systematic LND could possibly be omitted in patients without LNM. Therefore, the purposes of the present study were to investigate the efficacy of prophylactic LND in ICC patients without LNM, and to evaluate which clinicopathological factors might be associated with the accurate determination of LNM status.

\section{Patients and Methods}

\section{Patients}

We retrospectively analyzed 64 patients with ICC who underwent resection in our institution from January 1995 to December 2013. ICC was defined as adenocarcinoma arising from the second or greater peripheral branches of the intrahepatic bile ducts.

Patients with combined hepatocellular carcinoma and cholangiocarcinoma were excluded from this study. Six patients underwent only laparotomy due to peritoneal dissemination, 2 patients were revealed to have para aortic lymph node metastasis, and 2 patients were not considered to have undergone radical surgery because of positive surgical margins by pathological examination. Five patients were lost to follow-up. Therefore, the 49 remaining patients who underwent curative resection were enrolled in the present study. Of the 49 patients who underwent curative resection, 38 patients underwent LND, and 11 did not [LND(-) group]. Of the 38 patients who underwent LND, 28 patients did not show histological LNM [LND(+)-LNM(-) group], and 10 patients had histological LNM $[\operatorname{LND}(+)-\operatorname{LNM}(+)]$. To evaluate the efficacy of prophylactic LND in ICC patients, we compared the disease-free survival (DFS) and overall survival (OS) rates between the LND(-) group and the $\operatorname{LND}(+)-\operatorname{LNM}(-)$ group. The study protocol was approved by the Clinical Research Echics Committee of our hospital. Written informed consent which permit was obtained from all patients in the study.

\section{Preoperative diagnosis}

Preoperative computed tomography (CT) was performed to 
Citation: Satoh D, Matsukawa H, Araki H, Shiozaki S. The Role of Prophylactic Lymph Node Dissection in Patients with Intrahepatic Cholangiocarcinoma. J Surgery. 2016;4(2): 5.

ISSN: 2332-4139

evaluate the location, size, and number of the tumors. In cases of lymph nodes measuring $>5 \mathrm{~mm}$ in diameter on CT, we considered the possibility of LNM and determined LND should be performed. Meanwhile, when LN size was $<5 \mathrm{~mm}$ on preoperative CT, the decision to perform LND was left to the surgeon's discretion.

\section{Surgical technique}

Patients were excluded from surgical resection in cases of peritoneal carcinomatosis or extrahepatic metastasis confirmed at frozen section during surgery. Routine bile duct resection was not performed with LND. LND of the regional LNs was classified according to the Japanese Society of Biliary Surgery (JSBS) classification [20]. We harvested the LNs of the hepatoduodenal ligament $(12 \mathrm{~h}, 12 \mathrm{a}, 12 \mathrm{p}, 12 \mathrm{~b})$, the proper artery (8), and the posterior surface of the pancreatic head (13) during the surgery. In cases of tumor located in the left lobe, we performed sampling dissection of the LNs of the left gastric artery (7), and the LNs along the lesser curvature of the stomach, and around the cardia in addition to the above-mentioned regional LND. The presence of LNM was not regarded as a contraindication to surgical resection.

\section{Statistics}

Continuous data were expressed as mean \pm SD and were compared using the Mann-Whitney $U$ test. Categorical data were assessed using the chi-squared test. Patient survival and recurrence rates were estimated by the Kaplan-Meier method, and differences between survival curves were tested by the log-rank test. Multivariate analysis was performed according to the Cox proportional hazards model. Statistical analysis was carried out using JMP software (version 9.0; SAS Institute, Inc, Cary, NC, USA).

\section{Results}

Patient Characteristics in the $\operatorname{LND}(-)$ and $\operatorname{LND}(+)-\operatorname{LNM}(-)$ groups The demographics and linicopathological characteristics for patients in the LND(-) and LND(+)-LNM(-) groups are displayed in Table 1. There were no significant differences in age, gender, value of indocyanine green retention at 15 minutes (ICG15), serum CA199 level, tumor number, proportion of tumor locations, histological tumor differentiation grade, vascular invasion, or pTNM stage between the two groups, while tumor size was significantly larger, and the percentage of patients wth LN swelling $>5 \mathrm{~mm}$ on preoperative CT was higher in the $\mathrm{LND}(+)-\mathrm{LNM}(-)$ group than in the the $\mathrm{LND}(-)$ group.

\section{Analysis of OS and DFS in the LND(-) and LND(+)-LNM(-) groups}

The 1-, 3- and 5-year OS rates were 100.0, 80.0, and $64.6 \%$, respectively, in the $\mathrm{LND}(-)$ group, and $88.6,58.1$, and $45.8 \%$, respectively, in the $\mathrm{LND}(+)-\mathrm{LNM}(-)$ group. There was no significant difference in OS rates between the two groups $(p=0.353)$ (Figure 1). The 1-, 3- and 5-year DFS rates were 63.6, 50.9, and 33.9\%, respectively, in the $\mathrm{LND}(-)$ group, and 54.6, 45.3, and $45.3 \%$ respectively, in the LND(+)-LNM(-) group. There was also no significant difference in DFS rates between the two groups $(p=0.863)$ (Figure 1).

\section{Sites of initial recurrence}

In the $\mathrm{LND}(-)$ group, recurrence occurred in 6 of 11 patients; in
Table 1: Demographics and clinicopathological characteristics.

\begin{tabular}{|c|c|c|c|c|}
\hline & \multicolumn{2}{|r|}{ LND(-) } & \multirow{2}{*}{$\begin{array}{c}\text { LND(+)-LNM(-) } \\
(n=28)\end{array}$} & \multirow[t]{2}{*}{$p$ value } \\
\hline & & $(n=11)$ & & \\
\hline Age (years) & \multicolumn{2}{|c|}{$65.9 \pm 2.9$} & $67.8 \pm 1.8$ & 0.590 \\
\hline Gender (male/female) & & $5 / 6$ & $13 / 15$ & 0.956 \\
\hline ICG15 (\%) & \multicolumn{2}{|c|}{$8.3 \pm 2.1$} & $9.7 \pm 1.1$ & 0.717 \\
\hline CA19-9 (U/mL) & \multicolumn{2}{|c|}{$24.5 \pm 483.5$} & $607.6 \pm 299.9$ & 0.312 \\
\hline Tumor size (cm) & \multicolumn{2}{|c|}{$3.8 \pm 0.8$} & $6.0 \pm 0.5$ & 0.031 \\
\hline Tumor number & & & & 0.133 \\
\hline Single & & 11 & 23 & \\
\hline Multiple & & 0 & 5 & \\
\hline Location of tumor & & & & 0.243 \\
\hline Right lobe & & 4 & 16 & \\
\hline Left lobe & & 7 & 12 & \\
\hline LN swelling ( $\geq 5 \mathrm{~mm}$ ) & & $0(0 \%)$ & $8(28.6 \%)$ & 0.014 \\
\hline on preoperative $\mathrm{CT}$, & $\mathrm{n}$ & & & \\
\hline \multicolumn{5}{|l|}{ (\%) } \\
\hline Differentiation, n (\%) & & & & 0.989 \\
\hline Well & 3 & $(27.2 \%)$ & $8(28.6 \%)$ & \\
\hline Moderate & 5 & $(45.6 \%)$ & $13(46.4 \%)$ & \\
\hline Poor & 3 & $(27.2 \%)$ & $7(25.0 \%)$ & \\
\hline $\begin{array}{l}\text { Vascular invasion, } \\
\text { n (\%) }\end{array}$ & 2 & $(18.1 \%)$ & $6(21.4 \%)$ & 0.820 \\
\hline Satellite nodule, n (\%) & 6 & $(54.5 \%)$ & $22(78.6 \%)$ & 0.085 \\
\hline pTNM stage & & & & 0.218 \\
\hline I/II/III/IVa & & $2 / 3 / 5 / 1$ & & $1 / 3 / 18 / 6$ \\
\hline
\end{tabular}

CA19-9: Carbohydrate 19-9; LN: Lymph Node; LND: Lymph Node Dissection; LNM: Lymph Node Metastasis; CT: Computed Tomography; TNM: Tumor-NodeMetastasis
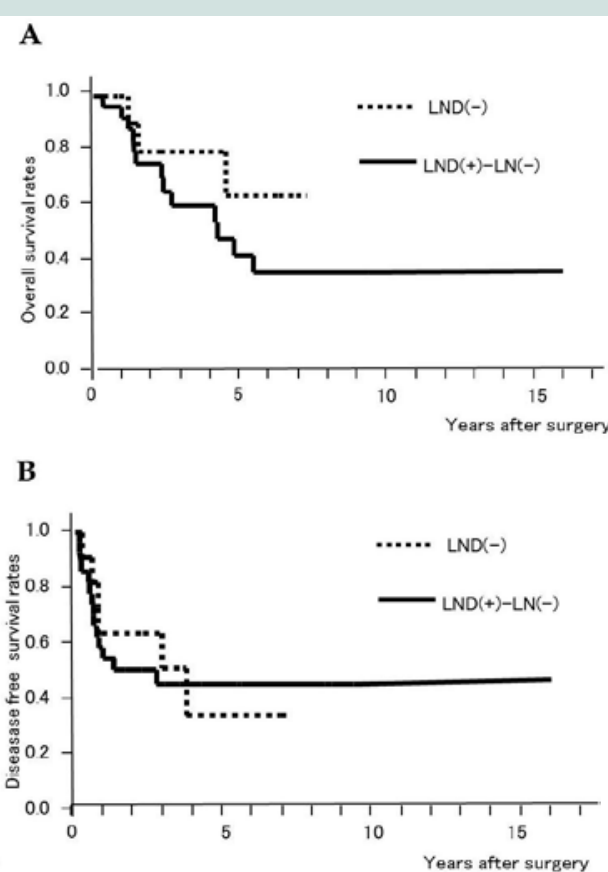

Figure 1: Overall survival and disease-free survival curves. (A) Survival curves of the LND(-) and LND(+)-LNM(-) groups. No significant difference was evident between the two groups $(p=0.353)$. (B) Disease-free survival curves of LND(-) and LND(+)-LNM(-) groups. No significant difference was evident between the two groups $(p=0.8634)$.

LN: Lymph Node; LND: Lymph Node Dissection 
Citation: Satoh D, Matsukawa H, Araki H, Shiozaki S. The Role of Prophylactic Lymph Node Dissection in Patients with Intrahepatic Cholangiocarcinoma. J Surgery. 2016;4(2): 5.

the $\mathrm{LND}(+)-\mathrm{LNM}(-)$ group, recurrence occurred in 14 of 28 patients (Table 2). Although no local LN recurrence occurred in the LND() group, local LN recurrence was seen in 2 patients of the $\operatorname{LND}(+)$ LNM(-) group. The most frequent recurrence site was the liver in both groups.

\section{Uni- and multi-variate risk factor analysis for histological lymph node metastasis}

Of the 38 patients who underwent LND, 10 patients had histological LNM. To identify risk factors for histological LNM, we performed uni- and multivariate analysis on preoperative data from the patients who underwent LND $(\mathrm{n}=38)$. Univariate analysis revealed that serum CA19-9 levels $>150 \mathrm{U} / \mathrm{mL}$, which was a cut-off value calculated by receiver operating characteristic (ROC) analysis $(p=0.001)$, and LN size $>5 \mathrm{~mm}$ on preoperative CT $(p=0.0005)$ were the significant risk factors for histological LNM (Table 3). Multivariate analysis revealed that serum CA19-9 levels $>150 \mathrm{U} / \mathrm{mL}$ $(p=0.005)$ and LN size $>5 \mathrm{~mm}$ on preoperative CT $(p=0.017)$ were the significant risk factors for histological LNM (Table 3)

\section{Association with the incidence of LNM and risk factors for LNM}

We evaluated the incidence of histological LNM, dividing the patients into 4 groups by LN size ( $5 \mathrm{~mm}$ ) and serum level of CA19-9 $(150 \mathrm{U} / \mathrm{mL})$ as preoperative independent risk factors of histological LNM (Table 4). It was revealed that none of the patients with LN size $<5 \mathrm{~mm}$ in preoperative CT and serum CA19-9 level $\leq 150 \mathrm{U} / \mathrm{mL}$ had histological LNM among the ICC patients who underwent LND. Conversely, $78 \%$ of the patients with LN size $>5 \mathrm{~mm}$ on preoperative CT and serum CA19-9 level $>150 \mathrm{U} / \mathrm{mL}$ showed histological LNM (Figure 2).

\section{Discussion}

Although the incidence of LNM in ICC patients is high, reportedly ranging from 36 to $62 \%$ [21-31], and LNM is a strong predictor of worse long-term outcome after curative-intent resection of ICC, the role of LND is still controversial. Some authors determine stage and to guide perioperative management [32]. Meanwhile, other authors have noted that LND was not effective in improving survival rates, and have recommended against routine LND [33,34]. Furthermore, as for prophylactic LND performed in patients without LNM, the

Table 2: Initial site of recurrence.

\begin{tabular}{|l|c|c|}
\hline & \multicolumn{2}{|l|}{$\begin{array}{l}\text { LND(-) } \\
(n=11)\end{array}$} \\
\hline Liver & 3 & 3 \\
\hline Liver and no. 16 LN & 1 & 1 \\
\hline Peritoneum & 0 & 3 \\
\hline Lung & 1 & 1 \\
\hline Bone & 1 & 3 \\
\hline Lung and bone & 0 & 1 \\
\hline Local LN & 0 & 2 \\
\hline Total & $6(54.5 \%)$ & $14(50.0 \%)$ \\
\hline
\end{tabular}

LN: Lymph Node; LND: Lymph Node Dissection; LNM: Lymph Node Metastasi
Table 3: Uni- and multivariate analysis of preoperative risk factors for histological LNM.

\begin{tabular}{|c|c|c|c|c|c|c|}
\hline \multirow[t]{2}{*}{ Factors } & \multicolumn{2}{|c|}{$\begin{array}{l}\text { Univariate } \\
\text { analysis }\end{array}$} & \multirow[b]{2}{*}{$p$ value } & \multicolumn{2}{|c|}{$\begin{array}{l}\text { Multivariate } \\
\text { analysis }\end{array}$} & \multirow[b]{2}{*}{$p$ value } \\
\hline & OR & $95 \% \mathrm{Cl}$ & & OR & $95 \% \mathrm{Cl}$ & \\
\hline \multirow[t]{2}{*}{ Age $>65$ years } & 1.667 & $0.348-7.0$ & 0.510 & & & \\
\hline & & 558 & & & & \\
\hline \multirow[t]{2}{*}{ Male gender } & 2.692 & $0.611-14$ & 0.195 & & & \\
\hline & & 0.542 & & & & \\
\hline \multirow[t]{2}{*}{ CA19-9 > $150 \mathrm{U} / \mathrm{mL}$} & 14.667 & $2.825-11$ & 0.001 & 18.919 & $2.301-42$ & 0.005 \\
\hline & & 6.642 & & & 7.561 & \\
\hline \multirow[t]{2}{*}{ Multiple tumors } & 4.6 & $0.963-23$ & 0.056 & 4.931 & $0.451-12$ & 0.196 \\
\hline & & 0.638 & & & 0.183 & \\
\hline \multirow[t]{2}{*}{ Tumor size $\geq 5 \mathrm{~cm}$} & 1.8 & $0.410-8.0$ & 0.431 & & & \\
\hline & & 025 & & & & \\
\hline \multicolumn{7}{|l|}{ Tumor location } \\
\hline \multirow[t]{2}{*}{ Left lobe } & 3.111 & $0.705-16$ & 0.136 & & & \\
\hline & & 0.862 & & & & \\
\hline \multirow[t]{2}{*}{ Hilar } & 3.6 & $0.537-71$ & 0.206 & & & \\
\hline & & 0.911 & & & & \\
\hline LN size $\geq 5 \mathrm{~mm}$ on & 22.5 & $3.434-45$ & 0.0005 & 13.985 & $1.548-34$ & 0.017 \\
\hline preoperative CT & & 1.177 & & & 0.965 & \\
\hline
\end{tabular}

CA19-9: Carbohydrate 19-9; LN: Lymph Node; LNM: Lymph Node Metastasis; CT: Computed Tomography

impact on outcome has not been elucidated at all.

The present retrospective study compared the OS and DFS rates between the patients who did not undergo LND and the patients who underwent LND but did not have LNM to clarify whether LND should be performed prophylactically in patients without LN involvement. The present study demonstrated that there was no significant difference between these two groups, which suggests that prophylactic LND does not contribute to either OS or DFS.

Prophylactic LND seems to prevent local LN recurrence by removal of possible microscopic LNM both around the perihepatic LNs and at frequent sites of recurrence. However, the present study investigated the site of initial recurrence, and revealed that local LN recurrence had occurred even in the patients who underwent regional LND despite the fact that none of the patients who did not undergo LND had local LN recurrence. It has been discovered that intrahepatic recurrence is a common recurrence pattern in both the patients who undergo LND and those who do not undergo LND as previous study reported [35,36]. Another study found that patients who underwent LND but had no LNM appeared to have slightly worse survival rates than patients who did not undergo LND during the earlier portion of the follow-up period [37]. Similarly, in the current study, the patients who underwent LND but had no LNM seemed to have slightly worse survival rates than the patients who did not undergo LND, although the difference was not statistically significant. There may have been some bias regarding whether LND was performed in the patients with LN size $<5 \mathrm{~mm}$ on preoperative CT.

If prophylactic LND could be omitted in the patients without 
Citation: Satoh D, Matsukawa H, Araki H, Shiozaki S. The Role of Prophylactic Lymph Node Dissection in Patients with Intrahepatic Cholangiocarcinoma. J Surgery. 2016;4(2): 5.

ISSN: 2332-4139

Table 4: Incidence of histological LNM.

\begin{tabular}{|l|l|l|}
\hline & Serum CA19-9 level & \\
\hline & $\leq 150 \mathrm{U} / \mathrm{mL}$ & $>150 \mathrm{U} / \mathrm{mL}$ \\
\hline LN size on preoperative CT & & \\
\hline$<5 \mathrm{~mm}$ & $0 / 16(0 \%)$ & $1 / 5(20 \%)$ \\
\hline$\geq 5 \mathrm{~mm}$ & $2 / 8(25 \%)$ & $7 / 9(78 \%)$ \\
\hline
\end{tabular}

CA19-9: Carbohydrate 19-9; LN: Lymph Node; LNM: Lymph Node Metastasis; CT: Computed Tomography

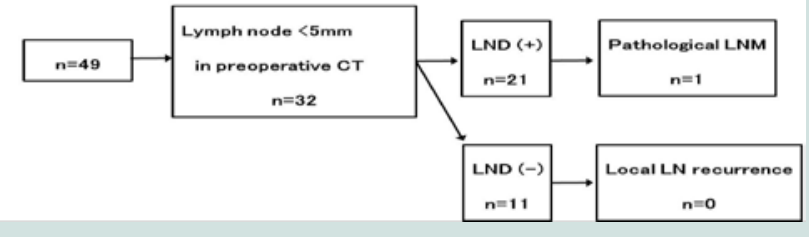

Figure 2: $\mathrm{LN}$ status in the patients with $\mathrm{LN}$ size $<5 \mathrm{~mm}$ in preoperative $\mathrm{CT}$. Of 32 patients with $\mathrm{LN}<5 \mathrm{~mm}$ in preoperative $\mathrm{CT}, 21$ patients underwent LND and pathological examination demonstrated that LNs were positive in only one patient. Among 11 patients who did not undergo LND, no patients had local $L N$ recurrence after curative resection.

CT: Computed Tomography; LN: Lymph Node; LND: Lymph Node Dissection; LNM: Lymph Node Metastasis

histological LNM, the problem is that preoperative diagnostic imaging techniques, including CT, MRI, and 18F - fluorodexyglucose positron emission tomography, would have difficulty in accurately detecting histological LNM preoperatively. Therefore, the present study investigated significant preoperative risk factors, not postoperative or histological factors, for histological LNM. The results revealed that LN size $>5 \mathrm{~mm}$ on preoperative CT and serum CA 19-9 level $>150$ $\mathrm{U} / \mathrm{mL}$ were significant preoperative predictors of histological LNM. In the patients with LN size $<5 \mathrm{~mm}$ on preoperative CT and serum.

CA19-9 level $\leq 150 \mathrm{U} / \mathrm{mL}$ among the patients who underwent LND, no patient had histological LNM. Conversely, in the patients with LN size $>5 \mathrm{~mm}$ on preoperative CT and serum CA19-9 level $>150 \mathrm{U} / \mathrm{mL}$ who showed histological LNM, the incidence of histological LNM was $78 \%$. These two factors can be very useful for accurately diagnosing LN status.

The present study had several limitations. The study was retrospective in nature, so there may have been some selection bias, especially in patients with LN size $<5 \mathrm{~mm}$ on preoperative CT, because the performance of LND was left to the surgeon's discretion in such patients. Another possible limitation was quite a small sample size in this study.

The small number of patients in some subsets may have resulted in a type error in assessing OS and DFS, so it is difficult to draw definite conclusion about the effect of prophylactic LND.

In conclusion, prophylactic LND did not improve survival significantly after curative-intent surgery for ICC, and did not prevent local recurrence in the present study. Systematic regional LND may be not suitable for patients with $\mathrm{LN}$ size $<5 \mathrm{~mm}$ on preoperative CT and serum CA19-9 level $\leq 150 \mathrm{U} / \mathrm{mL}$

\section{References}

1. Aljiffry M, Abdulelah A, Walsh M, Peltekian K, Alwayn I, et al. (2009) Evidence-based approach to cholangiocarcinoma: a systematic review of the current literature. J Am Coll Surg 208: 134-147.

2. Bektas H, Schrem H, Kleine M, Tamac A, Vondran FW, et al. (2013) Primary liver tumours-presentation, diagnosis and surgical treatment. In: Reeves $\mathrm{H}$, Manas DM, Lochan R (Eds), Liver tumors-epidemiology, diagnosis, prevention and treatment. InTech 91-116.

3. Ikai I, Arii S, Okazaki M, Okita K, Omata M, et al. (2007) Report of the 17th Nationwide Follow-Up Survey of Primary Liver Cancer in Japan. Hepatol Res 37: 676-691.

4. Endo I, Gonen M, Yopp AC, Dalal KM, Zhou Q, et al. (2008) Intrahepatic cholangiocarcinoma: rising frequency, improved survival, and determinants of outcome after resection. Ann Surg 248: 84-96.

5. Dodson RM, Weiss MJ, Cosgrove D, Herman JM, Kamel I, et al. (2013) Intrahepatic cholangiocarcinoma: management options and emerging therapies. J Am Coll Surg 217: 736-750.

6. Morimoto $\mathrm{Y}$, Tanaka Y, Ito T, Nakahara M, Nakaba H, et al. (2003) Long term survival and prognostic factors in the surgical treatment for intrahepatic cholangiocarcinoma. J Hepatobiliary Pancreat Surg 10: 432-440.

7. Konstadoulakis MM, Roayaie S, Gomatos IP, Labow D, Fiel MI, et al. (2008) Fifteen-year, single-center experience with the surgical management of intrahepatic cholangiocarcinoma: operative results and long-term outcome. Surgery 143: 366-374.

8. Choi SB, Kim KS, Choi JY, Park SW, Choi JS, et al. (2009) The prognosis and survival outcome of intrahepatic cholangiocarcinoma following surgical resection: association of lymph node metastasis and lymph node dissection with survival. Ann Surg Oncol 16: 3048-3056.

9. Nagorney DM, Donohue JH, Farnell MB, Schleck CD, Ilstrup DM (1993) Outcomes after curative resections of cholangiocarcinoma. Arch Surg 128: 871-877.

10. Kawarada Y, Yamagiwa K, Das BC (2002) Analysis of the relationships between clinicopathologic factors and survival time in intrahepatic cholangiocarcinoma. Am J Surg 183: 679-685.

11. Weber SM, Jarnagin WR, Klimstra D, DeMatteo RP, Fong Y, et al. (2001) Intrahepatic cholangiocarcinoma: resectability, recurrence pattern, and outcomes. J Am Coll Surg 193: 384-391.

12. Isaji S, Kawarada Y, Taoka H, Tabata M, Suzuki H, et al. (1999) Clinicopathological features and outcome of hepatic resection for intrahepatic cholangiocarcinoma in Japan. J Hepatobiliary Pancreat Surg 6: 108-116.

13. Morine Y, Shimada M, Utsunomiya T, Imura S, Ikemoto T, et al. (2012) Clinical impact of lymph node dissection in surgery for peripheral-type intrahepatic cholangiocarcinoma. Surg Today 42: 147-151.

14. Shimada M, Yamashita Y, Aishima S, Shirabe K, Takenaka K, et al. (2001) Value of lymph node dissection during resection of intrahepatic cholangiocarcinoma. Br J Surg 88: 1463-1466.

15. El Rassi ZE, Partensky C, Scoazec JY, Henry L, Lombard Bohas C, et al. (1999) Peripheral cholangiocarcinoma: presentation, diagnosis, pathology and management. Eur J Surg Oncol 25: 375-380.

16. Kluge R, Schmidt F, Caca K, Barthel H, Hesse S, et al. (2001) Positron emission tomography with [(18)F]fluoro-2-deoxy-D-glucose for diagnosis and staging of bile duct cancer. Hepatology 33: 1029-1035.

17. Seo S, Hatano E, Higashi T, Nakajima A, Nakamoto $Y$, et al. (2008) Fluorine-18 fluorodeoxyglucose positron emission tomography predicts lymph node metastasis, P-glycoprotein expression, and recurrence after resection in mass-forming intrahepatic cholangiocarcinoma. Surgery 143: 769-777.

18. Minagawa M, Ikai I, Matsuyama Y, Yamaoka Y, Makuuchi M (2007) Staging of hepatocellular carcinoma: assessment of the Japanese TNM and AJCC UICC TNM systems in a cohort of 13,772 patients in Japan. Ann Surg 245: 
Citation: Satoh D, Matsukawa H, Araki H, Shiozaki S. The Role of Prophylactic Lymph Node Dissection in Patients with Intrahepatic Cholangiocarcinoma. J Surgery. 2016;4(2): 5.

ISSN: 2332-4139

909-922.

19. Kenkyūkai NT (2004) Classification of biliary tract carcinoma. Japanese Society of Biliary Surgery ( $2^{\text {nd }}$ edn), Kanehara \& Co. Ltd, Tokyo, Japan.

20. Uenishi T, Hirohashi K, Kubo S, Yamamoto T, Yamazaki O, et al. (2001) Clinicopathological factors predicting outcome after resection of massforming intrahepatic cholangiocarcinoma. Br J Surg 88: 969-974.

21. Okabayashi T, Yamamoto J, Kosuge T, Shimada K, Yamasaki S, et al. (2001) A new staging system for mass-forming intrahepatic cholangiocarcinoma: analysis of preoperative and postoperative variables. Cancer 92: 2374-2383.

22. Inoue K, Makuuchi M, Takayama T, Torzilli G, Yamamoto J, et al. (2000) Long-term survival and prognostic factors in the surgical treatment of massforming type cholangiocarcinoma. Surgery 127: 498-505.

23. Kawarada Y, Yamagiwa K, Das BC (2002) Analysis of the relationships between clinicopathologic factors and survival time in intrahepatic cholangiocarcinoma. Am J Surg 183: 679-685.

24. Isa T, Kusano T, Shimoji H, Takeshima Y, Muto Y, et al. (2001) Predictive factors for long-term survival in patients with intrahepatic cholangiocarcinoma. Am J Surg 181: 507-511.

25. Shirabe K, Shimada M, Harimoto N, Sugimachi K, Yamashita Y, et al. (2002) Intrahepatic cholangiocarcinoma: its mode of spreading and therapeutic modalities. Surgery 131(1 Suppl): S159-S164.

26. Suzuki S, Sakaguchi T, Yokoi Y, Okamoto K, Kurachi K, et al. (2002) Clinicopathological prognostic factors and impact of surgical treatment of mass-forming intrahepatic cholangiocarcinoma. World J Surg 26: 687-693.

27. Uenishi T, Yamazaki O, Yamamoto T, Hirohashi K, Tanaka H, et al. (2005) Serosal invasion in TNM staging of mass-forming intrahepatic cholangiocarcinoma. J Hepatobiliary Pancreat Surg 12: 479-483.

28. Morimoto Y, Tanaka Y, Ito T, Nakahara M, Nakaba H, et al. (2003) Long- term survival and prognostic factors in the surgical treatment for intrahepatic cholangiocarcinoma. J Hepatobiliary Pancreat Surg 10: 432-440.

29. Nakagawa T, Kamiyama T, Kurauchi N, Matsushita M, Nakanishi K, et al. (2005) Number of lymph node metastases is a significant prognostic factor in intrahepatic cholangiocarcinoma. World J Surg 29: 728-733.

30. Ohtsuka M, Ito H, Kimura F, Shimizu H, Togawa A, et al. (2002) Results of surgical treatment for intrahepatic cholangiocarcinoma and clinicopathological factors influencing survival. Br J Surg 89: 1525-1531.

31. Ercolani G, Grazi GL, Ravaioli M, Grigioni WF, Cescon M, et al. (2004) The role of lymphadenectomy for liver tumors: further considerations on the appropriateness of treatment strategy. Ann Surg 239: 202-209.

32. Shirabe K, Mano Y, Taketomi A, Soejima Y, Uchiyama H, et al. (2010) Clinicopathological prognostic factors after hepatectomy for patients with mass-forming type intrahepatic cholangiocarcinoma: relevance of the lymphatic invasion index. Ann Surg Oncol 17: 1816-1822.

33. Li DY, Zhang HB, Yang N, Quan Y, Yang GS (2013) Routine lymph node dissection may be not suitable for all intrahepatic cholangiocarcinoma patients: results of a monocentric series. World J Gastroenterol 19: 90849091.

34. Miwa S, Miyagawa S, Kobayashi A, Akahane Y, Nakata T, et al. (2006) Predictive factors for intrahepatic cholangiocarcinoma recurrence in the liver following surgery. J Gastroenterol 41: 893-900

35. Nakagohri T, Asano T, Kinoshita H, Kenmochi T, Urashima T, et al. (2003) Aggressive surgical resection for hilar-invasive and peripheral intrahepatic cholangiocarcinoma. World J Surg 27: 289-293.

36. Choi SB, Kim KS, Choi JY, Park SW, Choi JS, et al. (2009) The prognosis and survival outcome of intrahepatic cholangiocarcinoma following surgical resection: association of lymph node metastasis and lymph node dissection with survival. Ann Surg Oncol 16: 3048-3056.

\section{Acknowledgements}

Study design: Satoh, Matuskawa. Acquisition of data: Shiozaki. Drafting of manuscript: Satoh, Manuscript editing: Shiozaki. 